\title{
Fuzzy Medial Ideals of BCl-Algebras with Interval-Valued Membership Function
}

\author{
S. M. Mostafa, \\ Department of \\ Mathematics, Faculty \\ of Education, Ain \\ Shams University, \\ Roxy, Cairo, Egypt.
}

\author{
A. E. Radwan, \\ Department of \\ Mathematics, Faculty \\ of science, Ain \\ Shams University, \\ Cairo, Egypt
}

\author{
A. M. Menshawy , \\ Department of \\ Mathematics, Faculty \\ of Education, Ain \\ Shams University, \\ Roxy, Cairo, Egypt.
}

\author{
R. Ghanem \\ Department of \\ Mathematics, Faculty \\ of Education, Ain \\ Shams University, \\ Roxy, Cairo, Egypt
}

\begin{abstract}
In this paper the notion of interval-valued Fuzzy medialideals (briefly i-v fuzzy medial -ideal) in BCI-algebras is introduced. Several theorems are stated and proved. The image and pre-image of interval-valued fuzzy medial -ideals are defined and how the homomorphic images and pre-images of interval-valued fuzzy medial -ideals become intervalvalued fuzzy medial -ideals in BCI-algebras is studied as well.
\end{abstract}

\section{Keywords}

Fuzzy medial-ideals- interval valued fuzzy medial-idealsimages and pre- images of interval valued fuzzy medial-ideals

AMS Subject Classification: 06F35, 03G25, 08A72

\section{INTRODUCTION}

The notion of BCK-algebras was proposed by Iseki[2,3,5]in 1966. In [4] , Iseki introduced the notion of a BCI-algebra which is a generalization of BCK-algebra. Since then numerous mathematical papers have been written investigating the algebraic properties of the BCK / BCIalgebras and their relationship with other structures including lattices and Boolean algebras. There is a great deal of literature which has been produced on the theory of $\mathrm{BCK} / \mathrm{BCI}$-algebras, in particular, emphasis seems to have been put on the ideal theory of BCK/BCI-algebras. For the general development of BCK/BCI-algebras the ideal theory plays an important role. The concept of fuzzy sets was first introduced by Zadeh [10] . From that time, the theory of fuzzy sets which has been developed in many directions and found applications in a wide variety of fields. In 1991, Xi [9] applied the concept of fuzzy sets to BCI, BCK, MV-algebras. The ideal theory and its fuzzification play an important role .In [7] J.Meng and Y.B.Jun studied medial BCI-algebras. In [8] S.M.Mostafa, Y.B.Jun and EL-menshawy introduce the notion of medial ideals in BCI-algebras, they state the fuzzification of medial ideals and investigate its properties In [11], Zadeh made an extension of the concept of fuzzy set by an interval-valued fuzzy set (i.e., a fuzzy set with an intervalvalued membership function). In [1], Biswas defined intervalvalued fuzzy subgroups and investigated some elementary properties.

In this paper, we use the notion of interval-valued fuzzy set by Zadeh to introduce the concept of an interval-valued fuzzy medial-ideal of BCI-algebras. Then we state and prove some theorems which determine the relationship between these conceps and BCI-sub-algebras. We give relations between interval-valued fuzzy subalgebras and interval-valued fuzzy medial- ideals. Moreover, We prove that every interval- valued fuzzy medial -ideal of a BCI-algebra $\mathrm{X}$ can be realized as medial -ideal of $X$. In connection with the notion of homomorphism, we study how the images and pre- images of interval-valued fuzzy medial -ideal become interval-valued fuzzy medial -ideal in BCI-algebra .

\section{PRELIMINARIES AND NOTATIONS}

In this section, we review some definitions and properties that will be useful in our results.

Definition 2.1. [4] An algebraic system $(X, *, 0)$ of type (2, 0 ) is called a BCI-algebra if it satisfying the following conditions:

$$
\begin{aligned}
& \text { (BCI-1) }((x * y) *(x * z)) *(z * y)=0, \\
& \text { (BCI-2) }(x *(x * y)) * y=0 \text {, } \\
& \text { (BCI-3) } x * x=0 \text {, } \\
& \text { (BCI-4) } x * y=0 \text { and } y * x=0 \text { imply } x=y \text {. }
\end{aligned}
$$

For all $x, y$ and $z \in X$. In a BCI-algebra $X$, we can define a partial ordering" $\leq$ " by $x \leq y$ if and only if $x * y=0$.

In what follows, $\mathrm{X}$ will denote a BCI-algebra unless otherwise specified.

Definition 2.2.[7]. A BCI-algebra $(X, *, 0)$ of type $(2,0)$ is called a medial BCI-algebra if it satisfying the following condition: $(x * y) *(z * u)=(x * z) *(y * u)$,for all $x, y, z$ and $u \in X$

Lemma 2.3.[7].An algebra $(X, *, 0)$ of type $(2,0)$ is a medial BCI-algebra if and only if it satisfies the following conditions:

$$
\begin{array}{ll}
\text { (i) } & x *(y * z)=z *(y * x) \\
\text { (ii) } & x * 0=x \\
\text { (iii) } & x * x=0 .
\end{array}
$$

Lemma 2.4.[7]. In a medial BCI-algebra $X$, the following holds:

$$
x *(x * y)=y, \text { for all } x, y \in X
$$


Lemma 2.5. Let $X$ be a medial BCI-algebra, then $0 *(y * x)=x * y$, for all $x, y \in X$

Proof. It is cleared.

Definition 2.6. A non empty subset $\mathrm{S}$ of a medial BCI-algebra $\mathrm{X}$ is said to be medial sub-algebra of $\mathrm{X}$, if $x * y \in S$, for all $x, y \in S$

Definition 2.7.[4]. A non-empty subset I of a BCI-algebra X is said to be a BCI-ideal of $\mathrm{X}$ if it satisfies:

(I1) $0 \in I$,

(I2) $x * y \in I$ and $y \in I$ implies $x \in I$ for all $x, y \in X$

Definition 2.8.[8]A non empty subset $\mathrm{M}$ of a medial BCIalgebra $X$ is said to be a medial ideal of $X$ if it satisfies:

$$
\text { (M1) } 0 \in M \text {, }
$$$$
\text { (M2) } z *(y * x) \in M \text { and } y * z \in M \text { imply } x \in M
$$
for all $x, y$ and $z \in X$

Proposition 2.9.[8]. Any medial ideal of a BCI-algebra must be a BCI- ideal but the converse is not true .

Proposition 2.10. Any BCI- ideal of a medial -BCI-algebra is a medial-ideal.

Proof. It is cleared

Example 2.11. Let $X=\{0,1,2,3,4,5\}$ be a set with a binary operation * defined by the following table:

\begin{tabular}{|l|l|l|l|l|l|l|}
\hline$*$ & 0 & 1 & 2 & 3 & 4 & 5 \\
\hline 0 & 0 & 0 & 0 & 0 & 4 & 4 \\
\hline 1 & 1 & 0 & 1 & 0 & 4 & 4 \\
\hline 2 & 2 & 2 & 0 & 0 & 4 & 4 \\
\hline 3 & 3 & 2 & 1 & 0 & 4 & 4 \\
\hline 4 & 4 & 4 & 4 & 4 & 0 & 0 \\
\hline 5 & 5 & 4 & 5 & 4 & 1 & 0 \\
\hline
\end{tabular}

Using the algorithms in Appendix B, we can prove that $(X, *, 0)$ is a BCI-algebra and

$\mathrm{A}=\{0,1,2,3\}$ is a medial-ideal of $\mathrm{X}$.

Definition 3.1.[6]. Let ${ }^{\mu}$ be a fuzzy set on a BCI-algebra $\mathrm{X}$, then ${ }^{\mu}$ is called a fuzzy BCI-subalgebra of $\mathrm{X}$ if :

$$
\mu(x * y) \geq \min \{\mu(x), \mu(y)\}, \text { for }
$$
$x, y \in X$

Definition 3.2 .[6].Let $X$ be a BCI-algebra. a fuzzy set ${ }^{\mu}$ in $\mathrm{X}$ is called a fuzzy $\mathrm{BCI}$-ideal of $\mathrm{X}$ if it satisfies:

$$
\text { (FI1) } \mu(0) \geq \mu(x) \text {, }
$$

(FI2) $\mu(x) \geq \min \{\mu(x * y), \mu(y)\}, \quad$ for all $x, y$ and $z \in X$

Definition 3.3.[8]. Let $X$ be a medial BCI-algebra. A fuzzy set ${ }^{\mu}$ in $\mathrm{X}$ is called a fuzzy medial ideal of $\mathrm{X}$ if it satisfies:

$$
\begin{aligned}
& \text { (FM1) } \mu(0) \geq \mu(x), \\
& \text { (FM2) } \mu(x) \geq \min \{\mu(z *(y * x)), \mu(y * z)\}, \text { for }
\end{aligned}
$$
all $x, y$ and $z \in X$.

Lemma 3.4: Any fuzzy medial-ideal of a BCI-algebra is a fuzzy BCI-ideal of X.

Proof. It is cleared.

Definition 3.5 . [6]. Let $\mathrm{f}$ be a mapping from the set $\mathrm{X}$ to a set Y. If ${ }^{\mu}$ is a fuzzy subset of $\mathrm{X}$, then the fuzzy subset $\mathrm{B}$ of $\mathrm{Y}$ defined by

$f(\mu)(y)=B(y)=\left\{\begin{array}{cc}\sup _{x \in f^{-1}(y)} \mu(x), & \text { if } f^{-1}(y)=\{x \in X, f(x)=y\} \neq \phi \\ 0 & \text { otherwise }\end{array}\right.$

Is called the image of ${ }^{\mu}$ under $\mathrm{f}$.

Similarly, if B is a fuzzy subset of $\mathrm{Y}$, then the fuzzy subset defined by $\mu(x)=B(f(x))$ for all $x \in X$, is said to be the preimage of $\mathrm{B}$ under $\mathrm{f}$.

Definition 3.6.[6]. Let $(X, *, 0)$ and $\left(Y, *^{\prime}, 0^{\prime}\right)$ be BCIalgebras. A mapping $f: X \rightarrow Y$ is said to be a homomorphism if $f(x * y)=f(x) *^{\prime} f(y)$ for all $x, y \in X$

\section{THE IMAGE AND THE PRE- IMAGE OF FUZZY MEDIAL IDEAL UNDER HOMOMORPHISM OF BCI- ALGEBRAS}

Theorem 3.7. An into homomorphic preimage of a fuzzy medial-ideal is also fuzzy medial-ideal.

Proof. Let $f: X \rightarrow X^{\prime}$ be an into homomorphism of BCI-algebras, B a fuzzy medial-ideal of $X^{\prime}$ and ${ }^{\mu}$ the preimage of B under $\mathrm{f}$, then $B(f(x))=\mu(x)$. For all $x \in X$

(FM1) hold, since

$\mu(0)=B(f(0)) \geq B(f(x))=\mu(x)$

Let

$x, y, z \in X$, then

$\mu(x)=B(f(x)) \geq \min \{B((f(z) *(f(y)) * f(x)), B(f(y) * f(z))\}$

$=\min \{B(f((z *(y * x)), B(f(y * z))\}=\min \{\mu((z *(y * x)), \mu(y * z)\}$.

Hence $\mu(x)=B(f(x))=(B \circ f)(x)_{\text {is }} \quad$ a fuzzy media-ideal of $\mathrm{X}$. The proof is completed. 
Theorem3.8. Let $f: X \rightarrow Y$ be a homomorphism between BCI-algebras X and Y. For every fuzzy medial-ideal $\mu_{\text {in } \mathrm{X},} f(\mu)$ is a fuzzy medial-ideal of $\mathrm{Y}$.

Proof. By definition,

$$
\begin{aligned}
& B\left(y^{\prime}\right)=f(\mu)\left(y^{\prime}\right):=\sup _{x \in f^{-1}\left(y^{\prime}\right)} \mu(x) \\
& \text { and } \sup \phi:=0
\end{aligned}
$$

We have to prove that $B\left(x^{\prime}\right) \geq \min \left\{B\left(\left(z^{\prime} *\left(y^{\prime} * x^{\prime}\right)\right), B\left(y^{\prime} * z^{\prime}\right)\right\}\right.$, for all $x^{\prime}, y^{\prime}, z^{\prime} \in Y$.

(i) Let $f: X \rightarrow Y$ be an onto homomorphism of BCI-algebras. Let $\mu$ be a fuzzy medial-ideal of $X$ with sup property and $B$ the image of $\mu$ under $f$. Since $\mu$ is a fuzzy medial-ideal of $X$, we have $\mu(0) \geq \mu(x)$, for all $x \in X$.

Note that $0 \in f^{-1}\left(0^{\prime}\right)$, where 0 and $0^{\prime}$ are the zeroes elements of $\mathrm{X}$ and $\mathrm{Y}$ respectively. Thus, $B\left(0^{\prime}\right)=\sup _{t \in f^{-1}\left(0^{\prime}\right)} \mu(t)=\mu(0) \geq \mu(x)$,

for all $x \in X$, which implies that $B\left(0^{\prime}\right) \geq \sup \mu(t)=B\left(x^{\prime}\right)$, $\operatorname{tof}_{t \in f^{-1}\left(x^{\prime}\right)} \mu(t)=B\left(x^{\prime}\right)$, for any

$\begin{array}{lll}\text { For } & x^{\prime}, y^{\prime}, z^{\prime} \in Y, & \text { let } \\ x_{0} \in f^{-1}\left(x^{\prime}\right) & , \quad y_{0} \in f^{-1}\left(y^{\prime}\right), & z_{0} \in f^{-1}\left(z^{\prime}\right)\end{array}$

be Such that

$\mu\left(x_{0}\right)=\sup _{t \in f^{-1}\left(x^{\prime}\right)} \mu(t), \mu\left(y_{0}\right)=\sup _{t \in f^{-1}\left(y^{\prime}\right)} \mu(t)$

and

$\mu\left(z_{0}\right)=\sup _{t \in f^{-1}\left(z^{\prime}\right)} \mu(t)$,hence

$\mu\left(\left(z_{0} *\left(y_{0} * x_{0}\right)\right)=B\left\{f\left(\left(z_{0} *\left(y_{0} * x_{0}\right)\right)\right\}=B\left(B\left(\left(z^{\prime} *\left(y^{\prime} * x^{\prime}\right)\right)\right.\right.\right.\right.$

$=\sup _{\left(z_{0} *\left(y_{0} * x_{0}\right) \in f^{-1}\left(z^{\prime} *\left(y^{\prime} * x^{\prime}\right)\right)\right.} \mu\left(\left(z_{0} *\left(y_{0} * x_{0}\right)\right)\right\}$

And then

$B\left(x^{\prime}\right)=\sup _{t \in f^{-1}\left(x^{\prime}\right)} \mu(t)=\mu\left(x_{0}\right)$

$\geq \min \left\{\mu\left(\left(\left(z_{0} *\left(y_{0} * x_{0}\right), \mu\left(y_{0} * z_{0}\right)\right\}=\right.\right.\right.$

$\min \left\{\sup _{t \in f^{-1}\left(\left(z^{\prime} *\left(y^{\prime} * x^{\prime}\right)\right)\right.} \mu(t), \sup _{t \in f^{-1}\left(y^{\prime} * z^{\prime}\right)} \mu(t)\right\}$

$\min \left\{B\left(\left(z^{\prime} *\left(y^{\prime} * x^{\prime}\right)\right), B\left(y^{\prime} * z^{\prime}\right)\right\}\right.$

Hence B is a fuzzy medial-ideal of Y. (ii) If $\mathrm{f}$ is not onto. For every $x^{\prime} \in Y$ we define $X_{x^{\prime}}:=f^{-1}\left(x^{\prime}\right)$. Since $\mathrm{f}$ is a homomorphism We have $\left(X_{z^{\prime}}\left(X_{y^{\prime}} * X_{x^{\prime}}\right) \subset X_{\left(\left(z^{\prime} *\left(y^{\prime} * x^{\prime}\right)\right)\right.}\right.$

for all $x^{\prime}, y^{\prime}, z^{\prime} \in Y$. Let $x^{\prime}, y^{\prime}, z^{\prime} \in Y_{\text {be an arbitrary }}$ given. If $z^{\prime} *\left(y^{\prime} * x^{\prime}\right) \notin \operatorname{Im}(\mathrm{f})=\mathrm{f}(\mathrm{X})$,

then by definition

$\left.B\left(z^{\prime} *\left(y^{\prime} * x^{\prime}\right)\right)\right)=0$. But if $\left.z^{\prime} *\left(y^{\prime} * x^{\prime}\right)\right) \notin_{\mathrm{f}(\mathrm{X})}$ i.e. $X_{\left(z^{\prime} *\left(y^{\prime} * x^{\prime}\right)\right)}=\phi$, then by $(*)$ at least one of $x^{\prime}, y^{\prime}$ and $z^{\prime} \notin f(X)$ and hence $B\left(x^{\prime}\right) \geq 0=\min \left\{B\left(\left(z^{\prime} *\left(y^{\prime} * x^{\prime}\right)\right), B\left(y^{\prime} * z^{\prime}\right)\right\}\right.$

This completes the proof.

4. INTERVAL-VALUED FUZZY MEDIAL-IDEAL OF BCI-ALGEBRAS

In this section, we begin with the concepts of interval-valued fuzzy sets.

An interval number is $\widetilde{\boldsymbol{a}}=\left[\boldsymbol{a}^{L}, \boldsymbol{a}^{U}\right]$, where $0 \leq a^{L} \leq a^{U} \leq 1_{\text {.Let } \mathrm{D}[0,1]}$

denote the family of all closed subintervals of $[0,1]$, i.e.,

$D[0,1]=\left\{\tilde{a}=\left[a^{L}, a^{U}\right]: a^{L} \leq a^{U} \quad\right.$ for $\left.a^{L}, a^{U} \in I\right\}$

We define the operations $\leq, \geq,=$,rmin and rmax in case of two elements in $\mathrm{D}[0,1]$. We consider two elements $\widetilde{a}=\left[a^{L}, a^{U}\right]_{\text {and }} \tilde{b}=\left[b^{L}, b^{U}\right]_{\text {in } \mathrm{D}[0,1]}$

Then

1- $\tilde{a} \leq \tilde{b} \quad$ iff $a^{L} \leq b^{L}, a^{U} \leq b^{U}$;

${ }_{2-} \tilde{a} \geq \tilde{b} \quad$ iff $a^{L} \geq b^{L}, a^{U} \geq b^{U}$

${ }_{3-} \tilde{a}=\tilde{b} \quad$ iff $a^{L}=b^{L}, a^{U}=b^{U}$;

${ }_{4-} r \min \{\tilde{a}, \tilde{b}\}=\left[\min \left\{a^{L}, b^{L}\right\}, \min \left\{a^{U}, b^{U}\right\}\right]$;

${ }_{5-} r \max \{\tilde{a}, \tilde{b}\}=\left[\max \left\{a^{L}, b^{L}\right\}, \max \left\{a^{U}, b^{U}\right\}\right]$

Here we consider that $\tilde{0}=[0,0]$ as least element and $\tilde{1}=[1,1]_{\text {as greatest element. }}$

Let $\tilde{a}_{i} \in D[0,1]$,where $i \in \Lambda$.We define 


$$
\begin{aligned}
& r \inf _{i \in \Lambda} \tilde{a}_{i}=\left[\inf _{i \in \Lambda} a^{L_{i}}, \inf _{i \in \Lambda} a^{U}{ }_{i}\right] \\
& r \sup _{i \in \Lambda} \tilde{a}_{i}=\left[\sup _{i \in \Lambda} a^{L_{i}}, \sup _{i \in \Lambda} a_{i}{ }_{i}\right]
\end{aligned}
$$

An interval valued fuzzy set (briefly, i-v-f-set) $\tilde{\mu}$ on $X$ is defined as

$\tilde{\mu}=\left\{\left\langle x,\left[\mu^{L}(x), \mu^{U}(x)\right], x \in X\right\rangle\right\}$

where

$\tilde{\mu}: X \rightarrow D[0,1]_{\text {and }} \mu^{L}(x) \leq \mu^{U}(x)_{\text {,for all }}$

$x \in X$. Then the ordinary fuzzy sets $\mu^{L}: X \rightarrow[0,1]$ and $\mu^{U}: X \rightarrow[0,1]_{\text {are called a lower fuzzy set and an }}$ upper fuzzy set of $\tilde{\mu}$ respectively.

Definition 4.1 . [6].

An interval-valued (briefly $\mathrm{i}-\mathrm{v}$ ) fuzzy set $\mathrm{A}$ in $\mathrm{X}$ is called an interval-valued fuzzy BCI-sub algebra of $\mathrm{X}$ if $\tilde{\mu}_{A}(x * y) \geq r \min \left\{\tilde{\mu}_{A}(x), \tilde{\mu}_{A}(y)\right\}$

$x, y \in X$

for all

Example 4.2. Let $X=\{0,1,2,3\}$ be a set with a binary operation * define by the following table:

\begin{tabular}{|l|l|l|l|l|}
\hline$*$ & 0 & 1 & 2 & 3 \\
\hline 0 & 0 & 1 & 2 & 3 \\
\hline 1 & 1 & 0 & 3 & 2 \\
\hline 2 & 2 & 3 & 0 & 1 \\
\hline 3 & 3 & 2 & 1 & 0 \\
\hline
\end{tabular}

Using the algorithms in Appendix B, we can prove that $(X, *, 0)$ is a BCI-algebra. Define $\tilde{\mu}_{\mathrm{A}}$ (x) as follows:

$\tilde{\mu}_{\mathrm{A}}(\mathrm{x})= \begin{cases}{[0.3,0.9]} & \text { if } x=\{0,1\} \\ {[0.1,0.6]} & \text { otherwise }\end{cases}$ easy to check that $\mathrm{A}$ is an interval-valued fuzzy sub-algebra.

Lemma 4.3 . If A is an interval-valued fuzzy BCI-subalgebra of $X$, then $\tilde{\mu}_{A}(0) \geq \tilde{\mu}_{A}(x)$, for all $x \in X$.

Proof. For every $\quad x \in X$, we have $\tilde{\mu}_{A}(0)=\tilde{\mu}_{A}(x * x) \geq r \min \left\{\tilde{\mu}_{A}(x), \tilde{\mu}_{A}(x)\right\}_{=}$ $=\tilde{\mu}_{A}(x)$.

Lemma 4.4 . Let A be an interval-valued fuzzy BCIsubalgebra of $\mathrm{X}$. if there exist a sequence $\left\{X_{n}\right\}$ in $\mathrm{X}$ such $\lim _{n \rightarrow \infty} \tilde{\mu}_{A}\left(x_{n}\right)=[1,1]_{\text {, then }} \tilde{\mu}_{A}(0)=[1,1]_{[6] \text {. }}$
Definition 4.5: An interval-valued fuzzy set $A=\left\{\left(x, \tilde{\mu}_{A}(x)\right), x \in X\right\}$ in BCI-algebra $\mathrm{X}$ is called an interval-valued fuzzy medial-ideal (i-v fuzzy medial-ideal, in short) if it satisfies the following conditions:

(1) $\tilde{\mu}_{A}(0) \geq \tilde{\mu}_{A}(x)$,

(2) $\tilde{\mu}_{A}(x) \geq r \min \left\{\tilde{\mu}_{A}(z *(y * x)), \tilde{\mu}_{A}(y * z)\right\}$ , for all $x, y, z \in X$.

Example 4.6. Let $X=\{0,1,2,3\}$ as in example (4.2). Define $\tilde{\mu}_{\mathrm{A}(\mathrm{x}) \text { as follows: }}$

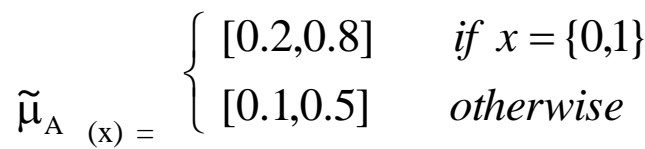
. It is easy to check that $\mathrm{A}$ is an interval-valued fuzzy medial-ideal of X.

Theorem 4.7: An interval-valued fuzzy set

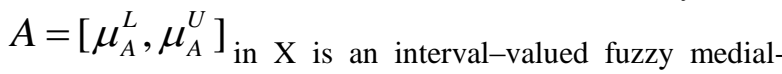
ideal of $\mathrm{X}$ if and only if $\mu_{A}^{L}$ and $\mu_{A}^{U}$ are fuzzy medial-ideal of X.

Proof. Let $\mu_{A}^{L}$ and $\mu_{A}^{U}$ are fuzzy medial-ideal of $\mathrm{X}$ and $x, y, z \in X$. Consider

$$
\begin{aligned}
& \tilde{\mu}_{A}(x)=\left[\mu_{A}^{L}(x), \mu_{A}^{U}(x)\right] \\
& \geq {\left[\min \left\{\mu_{A}^{L}(z *(y * x)), \mu_{A}^{L}(y * z)\right\}, \min \left\{\mu_{A}^{U}(z *(y * x)), \mu_{A}^{U}((y * z)\}\right]\right.} \\
&=r \min \left\{\left[\mu_{A}^{L}(z *(y * x)), \mu_{A}^{U}(z *(y * x))\right],\left[\mu_{A}^{L}(y * z), \mu_{A}^{U}(y * z)\right]\right\} \\
&=r \min \left\{\tilde{\mu}_{A}(z *(y * x)), \tilde{\mu}_{A}(y * z)\right\} .
\end{aligned}
$$

Conversely, suppose $\mathrm{A}$ is an interval-valued fuzzy medialideal of $\mathrm{X}$. For any $x, y, z \in X$ we have $\left[\mu_{A}^{L}(x), \mu_{A}^{U}(x)\right]=\tilde{\mu}_{A}(x) \geq$

$r \min \left\{\tilde{\mu}_{A}(z *(y * x)), \tilde{\mu}_{A}(y * z)\right\}=$

$=r \min \left\{\left[\mu_{A}^{L}(z *(y * x)), \mu_{A}^{U}(z *(y * x))\right],\left[\mu_{A}^{L}(y * z), \mu_{A}^{U}(y * z)\right]\right\}=$ $=\left[\min \left\{\mu_{A}^{L}(z *(y * x)), \mu_{A}^{L}(y * z)\right\}, \min \left\{\mu_{A}^{U}\left((z *(y * x)), \mu_{A}^{U}(y * z)\right\}\right]\right.$ .Therefore

$\mu_{A}^{L}(x) \geq \min \left\{\mu_{A}^{L}\left((z *(y * x)), \mu_{A}^{L}(y * z)\right\}\right.$ and

$\mu_{A}^{U}(x) \geq \min \left\{\mu_{A}^{U}(z *(y * x)), \mu_{A}^{U}(y * z)\right\}$.

Hence we get that $\mu_{A}^{L}$ and $\mu_{A}^{U}$ are fuzzy medial-ideal of X.

Theorem 4.8: Let A1and A2 be interval-valued fuzzy medial-ideals of $\mathrm{X}$. Then $A_{1} \cap A_{2}$ is an interval-valued fuzzy medial-ideal of $X$.

Proof.

$\tilde{\mu}_{A_{1} \cap A_{2}}(0)=\left[\mu_{A_{1} \cap A_{2}}^{L}(0), \mu_{A_{1} \cap A_{2}}^{U}(0)\right] \geq\left[\mu_{A_{1} \cap A_{2}}^{L}(x), \mu_{A_{1} \cap A_{2}}^{U}(x)\right]=\tilde{\mu}_{A_{1} \cap A_{2}}(x)$. 
Let $\quad x, y, z \in A_{1} \cap A_{2}$ be, such that $z *(y * x) \in\left\{A_{1}, A_{2}\right\}$ and $y * z \in\left\{A_{1}, A_{2}\right\}$. Since A1

and $\mathrm{A} 2$ are interval-valued fuzzy medial-ideals of $\mathrm{X}$, then by the theorem 4.7. We get

$\tilde{\mu}_{A_{1} \cap A_{2}}(x)=\left[\mu_{A_{1} \cap A_{2}}^{L}(x), \mu_{A_{1} \cap A_{2}}^{U}(x)\right]=\left[\min \left\{\mu_{A_{1}}^{L}(x), \mu_{A_{2}}^{L}(x)\right\}, \min \left\{\mu_{A_{1}}^{U}(x), \mu_{A_{2}}^{U}(x)\right\}\right]$ $\geq\left[\min \left\{\mu_{A_{1} \cap A_{2}}^{L}(z *(y * x)), \mu_{A_{1} \cap A_{2}}^{L}(y * z)\right\}, \min \left\{\mu_{A_{1} \cap A_{2}}^{U}(z *(y * x)), \mu_{A_{1} \cap A_{2}}^{U}(y * z)\right\}\right]$ $=r \min \left\{\tilde{\mu}_{A_{1} \cap A_{2}}(z *(y * x)), \tilde{\mu}_{A_{1} \cap A_{2}}(y * z)\right\}$.

Corollary 4.9: Let $\left\{A_{i} \mid i \in \Lambda\right\}$ be a family of interval$\bigcap A_{i}$

valued fuzzy medial-ideals of $X$. Then $i \in \Lambda$ is also an interval-valued fuzzy medial-ideal of X.

Definition 4.10. The non empty set $\tilde{U}\left(A ;\left[\delta_{1}, \delta_{2}\right]\right):\left\{x \in X \mid \tilde{\mu}_{A}(x) \geq\left[\delta_{1}, \delta_{2}\right]\right\}, \quad$ is called the interval-valued level fuzzy medial-ideal of $\mathrm{A}$, where $\left[\delta_{1}, \delta_{2}\right] \in D[0,1]$

Theorem 4.11: Let A be an interval-valued fuzzy set in $X$. Then $\mathrm{A}$ is an interval-valued fuzzy medial-ideal of $\mathrm{X}$ if and only if the non empty set $\tilde{U}\left(A ;\left[\delta_{1}, \delta_{2}\right]\right):\left\{x \in X \mid \tilde{\mu}_{A}(x) \geq\left[\delta_{1}, \delta_{2}\right]\right\} \quad$ is a medial-ideal of $\mathrm{X}$ for every $\left[\delta_{1}, \delta_{2}\right] \in D[0,1]$.

Proof. Assume that $\mathrm{A}$ is an interval-valued fuzzy medialideal of $\mathrm{X}$.Let $\left[\delta_{1}, \delta_{2}\right] \in D[0,1]$ be such that $z *(y * x), y * z \in \tilde{U}\left(A ;\left[\delta_{1}, \delta_{2}\right]\right)$ then $\tilde{\mu}_{A}(x) \geq r \min \left\{\tilde{\mu}_{A}(z *(y * x)), \tilde{\mu}_{A}(y * z)\right\} \geq$ $r \min \left\{\left[\delta_{1}, \delta_{2}\right],\left[\delta_{1}, \delta_{2}\right]\right\}=\left[\delta_{1}, \delta_{2}\right]$ and $x \in \tilde{U}\left(A ;\left[\delta_{1}, \delta_{2}\right]\right)$. Thus $\tilde{U}\left(A ;\left[\delta_{1}, \delta_{2}\right]\right)$ is medialideal of $\mathrm{X}$.

Conversely, assume that $\tilde{U}\left(A ;\left[\delta_{1}, \delta_{2}\right]\right)(\neq \phi)_{\text {is a }}$ medial-ideal of $\mathrm{X}$. For every $\left[\delta_{1}, \delta_{2}\right] \in D[0,1]$, suppose that there exist $x_{0}, y_{0}, z_{0} \in X$ such that

$\tilde{\mu}_{A}\left(x_{0}\right)<r \min \left\{\tilde{\mu}_{A}\left(z_{0} *\left(y_{0} * x_{0}\right), \tilde{\mu}_{A}\left(y_{0} * z_{0}\right)\right\}\right.$. Let $\tilde{\mu}_{A}\left(z_{0} *\left(y_{0} * x_{0}\right)\right)=\left[\gamma_{1}, \gamma_{2}\right]$

$\tilde{\mu}_{A}\left(y_{0} * z_{0}\right)=\left[\gamma_{3}, \gamma_{4}\right]$ and $\tilde{\mu}_{A}\left(x_{0}\right)=\left[\delta_{1}, \delta_{2}\right]$. Then $\left[\delta_{1}, \delta_{2}\right]<r \min \left\{\left[\gamma_{1}, \gamma_{2}\right],\left[\gamma_{3}, \gamma_{4}\right]\right\}$

Taking $\left[\lambda_{1}, \lambda_{2}\right]=\left[\frac{1}{2}\left\{\tilde{\mu}_{A}\left(x_{0}\right)+r \min \left\{\tilde{\mu}_{A}\left(\left(z_{0} *\left(y_{0} * x_{0}\right)\right), \tilde{\mu}_{A}\left(y_{0} * z_{0}\right)\right\}\right]\right\}\right.$

$$
\begin{aligned}
& \frac{1}{2}\left(\left[\delta_{1}, \delta_{2}\right]+\left\{\min \left\{\gamma_{1}, \gamma_{2}\right\}, \min \left\{\gamma_{3}, \gamma_{4}\right\}\right\}\right)= \\
& \frac{1}{2}\left[\left(\delta_{1}+\min \left\{\gamma_{1}, \gamma_{3}\right\}\right),\left(\delta_{2}+\min \left\{\gamma_{2}, \gamma_{4}\right\}\right)\right] . \text {.It } \\
& \text { follows } \min \left\{\gamma_{1}, \gamma_{3}\right\}>\lambda_{1}=\frac{1}{2} \\
& \left(\delta_{1}+\min \left\{\gamma_{1}, \gamma_{3}\right\}\right)>\delta_{1} \text { and } \\
& \min \left\{\gamma_{2}, \gamma_{4}\right\}>\lambda_{2}=\frac{1}{2}\left(\delta_{2}+\min \left\{\gamma_{2}, \gamma_{4}\right\}\right)>\delta_{2} \text {, that }
\end{aligned}
$$$$
\left.\left[\min \left\{\gamma_{1}, \gamma_{3}\right\}\right), \min \left\{\gamma_{2}, \gamma_{4}\right\}\right]>\left[\lambda_{1}, \lambda_{2}\right]>\left[\delta_{1}, \delta_{2}\right]=\tilde{\mu}_{A}\left(x_{0}\right)
$$$$
\text { . There fore } x_{0} \notin \tilde{U}\left(A ;\left[\lambda_{1}, \lambda_{2}\right]\right) \text {. }
$$

On the other hand $\tilde{\mu}_{A}\left(z_{0} *\left(y_{0} * x_{0}\right)\right)=\left[\gamma_{1}, \gamma_{2}\right] \geq$ $\left[\min \left\{\gamma_{1}, \gamma_{3}\right\}, \min \left\{\gamma_{2}, \gamma_{4}\right\}\right]>\left[\lambda_{1}, \lambda_{2}\right]$, $\tilde{\mu}_{A}\left(y_{0} * z_{0}\right)=\left[\gamma_{3}, \gamma_{4}\right]$ $\geq\left[\min \left\{\gamma_{1}, \gamma_{3}\right\}, \min \left\{\gamma_{2}, \gamma_{4}\right\}\right],\left[\lambda_{1}, \lambda_{2}\right]$, and so $\left.z_{0} *\left(y_{0} * x_{0}\right)\right), y_{0} * z_{0} \notin \tilde{U}\left(A ;\left[\lambda_{1}, \lambda_{2}\right]\right)$. It contradicts that $\tilde{U}\left(A ;\left[\lambda_{1}, \lambda_{2}\right]\right)$ is a medial-ideal of $\mathrm{X}$. Hence $\tilde{\mu}_{A}(x) \geq r \min \left\{\tilde{\mu}_{A}(z *(y * x)), \tilde{\mu}_{A}(y * z)\right\} \quad$ for $\quad$ all $x, y, z \in X$.

Theorem 4.12. Every medial-ideal of $X$ can be realized as an interval-valued level fuzzy medial-ideal of X.

Proof. Let $\mathrm{Y}$ be a medial-ideal of $\mathrm{X}$ and let $\mathrm{A}$ be an intervalvalued fuzzy set on $\mathrm{X}$ defined by

$\tilde{\mu}_{A}=\left\{\begin{array}{lr}{\left[\alpha_{1}, \alpha_{2}\right]} & \text { if } x \in Y \\ {[0,0]} & \text { otherwise }\end{array}\right.$,

where $\alpha_{1}, \alpha_{2} \in(0,1]_{\text {with }} \alpha_{1}<\alpha_{2}$.

It is clear that $\tilde{U}\left(A ;\left[\alpha_{1}, \alpha_{2}\right]\right)=Y$. We show that $\mathrm{A}$ is an interval-valued fuzzy medial-ideal of X. Let $x, y, z \in X$. If $z *(y * x), y * x \in Y$, then $x \in Y, \quad$ and $\quad$ so $\tilde{\mu}_{A}(x) \geq\left[\alpha_{1}, \alpha_{2}\right]=r \min \left\{\left[\alpha_{1}, \alpha_{2}\right],\left[\alpha_{1}, \alpha_{2}\right]\right\}=$ $r \min \left\{\tilde{\mu}_{A}(z *(y * x)), \tilde{\mu}_{A}(y * z)\right\}$.

$$
\begin{aligned}
& \text { If } z *(y * x), y * z \notin Y, \\
& \left.\tilde{\mu}_{A}(z *(y * x))=[0,0]=\tilde{\mu}_{A}(y * z)\right\} \text { and } \\
& \tilde{\mu}_{A}(x) \geq[0,0]=r \min \{[0,0],[0,0]\}= \\
& r \min \left\{\tilde{\mu}_{A}(z *(y * x)), \tilde{\mu}_{A}(y * z)\right\} . \\
& \text { If } \quad z *(y * x) \in Y \quad \text { then } \\
& \tilde{\mu}_{A}(x) \geq[0,0]=r \min \left\{\left[\alpha_{1}, \alpha_{1}\right],[0,0]\right\} \text { and }, \quad \text { then } \\
& \tilde{\mu}_{A}(x) \geq r \min \left\{\tilde{\mu}_{A}(z *(y * x)), \tilde{\mu}_{A}(y * z)\right\} . \\
& \text { Similarly for the case } z *(y * x) \notin Y \text { and } y * z \in Y \text {,we }
\end{aligned}
$$


get $\quad \tilde{\mu}_{A}(x) \geq r \min \left\{\tilde{\mu}_{A}(z *(y * x)), \tilde{\mu}_{A}(y * z)\right\}$

Therefore $\mathrm{A}$ is an interval-valued fuzzy medial-ideal of $\mathrm{X}$. This completes the proof

Theorem 4.13. Let $\mathrm{Y}$ be a subset of $\mathrm{X}$ and let $\mathrm{A}$ be an interval-valued fuzzy set on $X$ defined by $\tilde{\mu}_{A}=\left\{\begin{array}{lr}{\left[\alpha_{1}, \alpha_{2}\right]} & \text { if } x \in Y \\ {[0,0]} & \text { otherwise }\end{array}\right.$,

if $\mathrm{A}$ is an interval-valued fuzzy medial-ideal of $\mathrm{X}$, then $\mathrm{Y}$ is medial-ideal of $X$

Proof. Assume that $\mathrm{A}$ is an interval-valued fuzzy medialideal of $\mathrm{X}$, and let $z *(y * x), y * z \in Y$, then $\tilde{\mu}_{A}(z *(y * x))_{=}\left[\alpha_{1}, \alpha_{2}\right]_{=} \tilde{\mu}_{A}(y * z), \quad$ and so $\tilde{\mu}_{A}(x) \geq r \min \left\{\tilde{\mu}_{A}\left((z *(y * x)), \tilde{\mu}_{A}(y * z)\right\}\right.$ $r \min \left\{\left[\alpha_{1}, \alpha_{2}\right],\left[\alpha_{1}, \alpha_{2}\right]\right\}=\left[\alpha_{1}, \alpha_{2}\right]$, this implies that $x \in \mathrm{Y}$. Hence $\mathrm{Y}$ is medial-ideal of $\mathrm{X}$. This completes the proof.

Theorem 4.14: If $\mathrm{A}$ is an interval-valued fuzzy medial-ideal of $\mathrm{X}$, then the set $X_{\widetilde{\mu}_{A}}:=\left\{x \in X \mid \tilde{\mu}_{A}(x)=\tilde{\mu}_{A}(0)\right\}_{\text {is }}$ a medial-ideal of $X$.

Proof. Let $z *(y * x), y * z \in X_{\tilde{\mu}_{A}}$. Then $\tilde{\mu}_{A}\left((z *(y * x))=\tilde{\mu}_{A}(0)=\tilde{\mu}_{A}(y * z)\right.$, and so

$\tilde{\mu}_{A}(x) \geq r \min \left\{\tilde{\mu}_{A}\left((z *(y * x)), \tilde{\mu}_{A}(y * z)\right\}_{=}\right.$

$r \min \left\{\tilde{\mu}_{A}(0), \tilde{\mu}_{A}(0)\right\}=\tilde{\mu}_{A}(0)$

Combining this with condition (1) of definition 4.5. We get $\tilde{\mu}_{A}(x)=\tilde{\mu}_{A}(0)$, that is $x \in X_{\tilde{\mu}_{A}}$. Hence $X_{\tilde{\mu}_{A} \text { is a }}$ medial-ideal of $X$. This completes the proof.

Definition 4.15. [1].

Let $f: X \rightarrow Y$ be a mapping from set $\mathrm{X}$ into a set $\mathrm{Y}$. let B be interval-valued fuzzy set in Y. Then the inverse image of $\mathrm{B}$, denoted by $f^{-1}(B)$, is interval-valued fuzzy set in $\mathrm{X}$ with the membership function given by $\tilde{\mu}_{f^{-1}(B)}(x)=\tilde{\mu}_{B}(f(x))$, for all $x \in X$

Lemma 4.16 .[1].Let $\mathrm{f}$ be a mapping from set $\mathrm{X}$ into a set $\mathrm{Y}$ let $m=\left[m^{L}, m^{u}\right]$, and

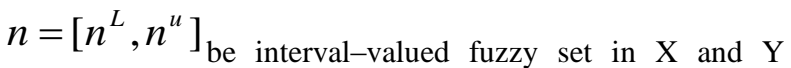
respectively. Then

$$
\begin{aligned}
& \text { (1) } f^{-1}(n)=\left[f^{-1}\left(n^{L}\right), f^{-1}\left(n^{u}\right)\right], \\
& \text { (2) } f(m)=\left[f\left(m^{L}\right), f\left(m^{u}\right)\right] .
\end{aligned}
$$

Theorem 4.17: Let $\mathrm{f}$ be homomorphism from a BCI-algebra $\mathrm{X}$ into a BCI-algebra Y. If $\widetilde{B}_{\text {is }}$ an interval-valued fuzzy medial-ideal of $\mathrm{Y}$, then the inverse image $f^{-1}(\tilde{B})$ of $\tilde{B}$ is interval-valued fuzzy medial-ideal of X.

Proof. Since $\tilde{B}=\left[\mu_{B}^{L}, \mu_{B}^{u}\right]_{\text {is an interval-valued fuzzy }}$ medial-ideal of Y, it follows that from theorem 4.7, that $\mu_{B}^{L}$ and $\mu_{B}^{u}$ are fuzzy medial-ideal of Y. hence by lemma 4.16 , we conclude that $f^{-1}(\widetilde{B})=\left[f^{-1}\left(\mu_{B}^{l}\right), f^{-1}\left(\mu_{B}^{u}\right)\right]_{\text {is an }}$ interval-valued fuzzy medial-ideal of $X$.

Theorem 4.18: Let $\mathrm{f}$ be a homomorphism from a BCIalgebra $\mathrm{X}$ into a BCI-algebra Y. If $\widetilde{A}_{\text {is an interval-valued }}$ fuzzy medial-ideal of $\mathrm{X}$, then $\mathrm{f}\left[\tilde{A}_{]}\right.$is an interval-valued fuzzy medial-ideal of $\mathrm{Y}$.

Proof. Assume that $\widetilde{A}=\left[\mu_{A}^{L}, \mu_{A}^{u}\right]$ is an interval-valued fuzzy medial-ideal of $X$. It follows from theorem 4.7 , that the images $f\left(\mu_{A}^{L}\right)$ and $f\left(\mu_{A}^{u}\right)$ are fuzzy medial-ideal of Y. Combining theorem 4.7, and lemma 4.16, we conclude that $f(\widetilde{A})=\left[f\left(\mu_{A}^{L}\right), f\left(\mu_{A}^{u}\right)\right]_{\text {is an an interval-valued fuzzy }}$ medial-ideal of $\mathrm{Y}$.

\section{CARTESIAN PRODUCT OF INTERVAL-VALUED FUZZY MEDIAL-IDEALS}

\section{Definition 5.1. [1].}

Let $\tilde{\mu}$ and $\tilde{\beta}$ be interval-valued fuzzy subset of a set $\mathrm{S}$, the Cartesian product of $\tilde{\mu}$ and $\tilde{\beta}$ is define by $(\tilde{\mu} \times \tilde{\beta}$ ) $(\mathrm{x}, \mathrm{y})=\mathrm{r} \min \left\{\tilde{\mu}_{(\mathrm{x}),} \tilde{\beta}_{(\mathrm{y})\}, \forall}{ }_{\mathrm{x}, \mathrm{y}} \in \mathrm{S}\right.$.

Remark: Let $\mathrm{X}$ and $\mathrm{Y}$ be BCI- algebras, we define $*$ on $\mathrm{X} \times$ $\mathrm{Y}$ as follows, for every

$(\mathrm{x}, \mathrm{y}),(\mathrm{u}, \mathrm{v}) \in \mathrm{X} \mathrm{x} \mathrm{Y},(\mathrm{x}, \mathrm{y}) *(\mathrm{u}, \mathrm{v})=(\mathrm{x} * \mathrm{u}, \mathrm{y} * \mathrm{v})$, then clearly $(\mathrm{X} \times \mathrm{Y}, *,(0,0))$ is a BCI- algebra.

Theorem 5.2: Let $\tilde{\mu}$ and $\tilde{\beta}$ be interval-valued fuzzy medial- ideals of BCI - algebra $\mathrm{X}$, then $\tilde{\mu} \times \tilde{\beta}$ is intervalvalued fuzzy medial- ideals of $\mathrm{X} \times \mathrm{X}$.

Proof : for any $(\mathrm{x}, \mathrm{y}) \in \mathrm{X} \times \mathrm{X}$, we have ,

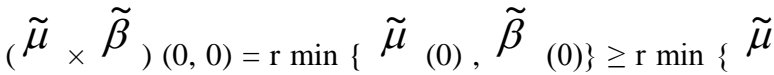

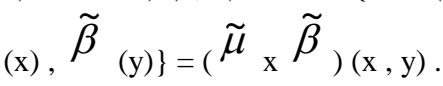

Now let $(\mathrm{x} 1, \mathrm{x} 2),(\mathrm{y} 1, \mathrm{y} 2),(\mathrm{z} 1, \mathrm{z} 2) \in \mathrm{X} \times \mathrm{X}$, then ,

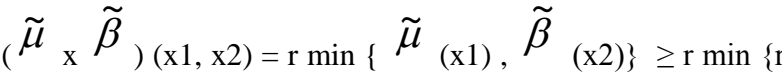

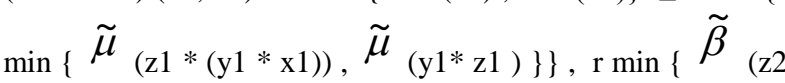

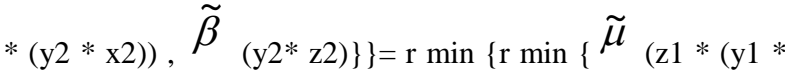

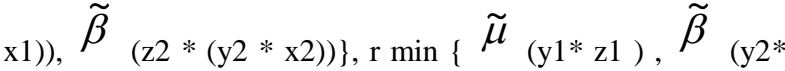


$\mathrm{z} 2)\}\}=\mathrm{r} \min \{(\tilde{\mu} \times \tilde{\beta})(\mathrm{z} 1 *(\mathrm{y} 1 * \mathrm{x} 1), \mathrm{z} 2 *(\mathrm{y} 2 * \mathrm{x} 2)),($ $\tilde{\mu}_{\times} \tilde{\beta}_{(\mathrm{y} 1 * \mathrm{z} 1, \mathrm{y} 2 * \mathrm{z} 2)\} \text {. Hence }} \tilde{\mu} \times \tilde{\beta}$ is intervalvalued a fuzzy medial- ideal of $\mathrm{X} \times \mathrm{X}$.

\section{CONCLUSIONS}

In the present paper, we have introduced the concept of interval-valued - fuzzy medial ideals of BCI-algebras and investigated some of their useful properties. We believe that these results are very useful in developing algebraic structures also these definitions and main results can be similarly extended to some other algebraic systems such as PS algebras , Q- algebras, SU- algebras , IS- algebras, $\beta$ algebras and semirings (hemirings). It is our hope that this work would other foundations for further study of the theory of BCI-algebras. In our future study of fuzzy structure of BCIalgebras, may be the following topics should be considered:

(1) To establish $\tilde{\tau}$ - interval-valued fuzzy medial -ideals of BCI-algebras;

(2) To consider the structure of $(\tilde{\tau}, \tilde{\rho})$ - interval-valued fuzzy medial -ideals of BCI-algebras

(3) To get more results in $\tilde{\tau}$ - cubic medial -ideals and it's application.

\section{ACKNOWLEDGEMENTS}

The authors would like to express sincere appreciation to the referees for their valuable suggestions and comments helpful in improving this paper.

\section{APPENDIX B. ALGORITHMS}

This appendix contains all necessary algorithms.

\subsection{Algorithm for BC I-Algebras}

Input ( $X:_{\text {set, }}{ }^{*}:_{\text {binary operation) }}$

Output (“ $X$ is a BCI -algebra or not”)

Begin

If $X=\phi$ then go to (1.);

End If

If $0 \notin X$ then go to (1.);

End If

Stop: =false;

$i:=1$,

While $i \leq|X|$ and not (Stop) do

If $x_{i} * x_{i} \neq 0$ then

Stop: $=$ true;

End If

$j:=1$
While $j \leq|X|$ and not (Stop) do

If $\left(x_{i} *\left(x_{i} * y_{j}\right)\right) * y_{j} \neq 0$, then

Stop: $=$ true

End If

End $\mathrm{f}$

$k:=1$

While $k \leq|X|$ and not (Stop) do

If $\left(\left(x_{i} * y_{j}\right) *\left(x_{i} * z_{k}\right)\right) *\left(z_{k} * y_{i}\right) \neq 0$, then

Stop: $=$ true;

End If

End If While

End If While

End If While

If Stop then

Output (“ $X$ is not a BCI-algebra”)

Else

Output (" $X$ is a BCI -algebra")

End If

End

\subsection{Algorithm for Fuzzy Subsets}

Input ( $X:{ }_{\text {BCI-algebra, }} \mu: X \rightarrow[0,1]$ );

Output (“ $A$ is a fuzzy subset of $X$ or not”)

Begin

Stop: =false;

$i:=1$;

While $i \leq|X|$ and not (Stop) do

If $\left(\mu\left(x_{i}\right)<0\right)$ or $\left(\mu\left(x_{i}\right)>1\right.$ ) then

Stop: $=$ true;

End If

End If While

If Stop then

Output (" $\mu$ is a fuzzy subset of $X$ ”)

Else

Output (“ $\mu^{\mu}$ is not a fuzzy subset of $X$ ”)

End If

End. 


\subsection{Algorithm for Medial -Ideals}

Input ( $X:$ BCI-algebra, $I$ : subset of $X$ );

Output (" $I$ is an medial -ideals of $X$ or not");

Begin

If $I=\phi$ then go to (1.);

End If

If $0 \notin I$ then go to (1.);

End If

Stop: =false;

$i:=1$

While $i \leq|X|$ and not (Stop) do

$j:=1$

While $j \leq|X|$ and not (Stop) do

$k:=1$

While $k \leq|X|$ and not (Stop) do

If $z_{k} *\left(y_{j} * x_{i}\right) \in I$ and $y_{j} * z_{k} \in I$ then

If $x_{i} \notin I$ then

Stop: $=$ true;

End If

End If

End If While

End If While

End If While
If Stop then

Output (" $I$ is is an medial -ideals of $X$ ")

Else (1.) Output (" $I$ is not is an medial -ideals of $X$ ”)

End If

End.

\section{REFERENCES}

[1] R. Biswas, Rosenfeld's fuzz subgroups with interval valued membership function, Fuzzy Sets and Systems 63 (1994), No. 1 87-90.

[2] M.Abe and K.Iseki, A survey on BCK and BCI algebras, Congresso de Logica Aplicada Techologia, LAPTEC 2000, 431-443. Festschrift in Hornor of Gabriel Thierrin, World Sci. (2001), 231-238.

[3] K. Iseki and S. Tanaka, An introduction to the theory of BCK-algebras, Math. Japon. 23(1978), 1-26.

[4] K. Iseki, On BCI-algebras, Math. Sem. Notes 8 (1980) 125-130.

[5] Y.Imai and K.Iseki: On axiom systems of Propositional calculi, XIV, Proc. Japan Acad. Ser A, Math Sci., 42(1966),19-22.

[6] Y. Bae Jun , VALUED FUZZY SUBALGEBRAS/IDEALS IN BCK-ALGEBRAS Scientiae Mathematicae Vol. 3, No. 3(2000), 435\{444

[7] J. Meng and Y.B. Jun, Notes on medial BCI-algebras, Comm. Korean Math. Soc. 8(1) (1993),33-37.

[8] S. M. Mostafa, Y. B. Jun and A. El-menshawy, Fuzzy medial ideals in BCI-algebras, fuzzy math., vol.7, no.2,1999, pp445-457.

[9] O. G. Xi, Fuzzy BCK-algebras, Math. Japon. 36(5) (1991), 935-942.

[10] L. A. Zadeh, Fuzzy sets, Information and Control 8 (1965) 338-353.

[11] L. A. Zadeh The concept of a linguistic variable and its application to approximate reasoning. I, Information Sci. 8 (1975), 199-249. MR 52\#7225a. Zbl 397.68071. 